\title{
Side Effects of Transcranial Magnetic Stimulation Biased Task Performance in a Cognitive Neuroscience Study
}

\author{
Birgit Abler*, Henrik Walter*, Arthur Wunderlich+, Jo Grothe*, Carlos Schönfeldt-Lecuona*, \\ Manfred Spitzer*, and Uwe Herwig*^
}

\begin{abstract}
Summary: Transcranial magnetic stimulation (TMS) is increasingly used as a research tool for functional brain mapping in cognitive neuroscience. Despite being mostly tolerable, side effects of TMS could influence task performance in behavioural TMS studies. In order to test this issue, healthy subjects assessed the discomfort caused by the stimulation during a verbal working memory task. We investigated the relation between subjective disturbance and task performance. Subjects were stimulated during the delay period of a delayed-match-to-sample task above cortical areas that had been identified before to be involved in working memory. Task performance and subjective disturbance due to side effects were monitored. The subjects' grade of discomfort correlated with the error rates: the higher the discomfort, the more errors were made. Conclusively, TMS side effects may bias task performance in cognitive neuroscience studies and may thereby lead to misinterpretation of results. We emphasize the importance of controlling side effects of the stimulation as a source of biasing effects in TMS studies.
\end{abstract}

Key words: Transcranial magnetic stimulation; TMS side effects; Cognitive neuroscience.

\section{Introduction}

Transcranial magnetic stimulation (TMS) has become an established tool for functional brain mapping purposes in cognitive neuroscience (Walsh and Cowey 2000). Although TMS is regarded as a mostly painless way of stimulating the brain through the scalp, it is not free of discomforting sensations. First, a clicking noise produced by the discharge of the stimulator through the coil is hard to mask even by ear-plugs. Second, TMS elicits a tactile sensation on the scalp due to stimulation of cutaneous nerves and contraction of scalp muscles under the coil. These sensations may disturb the subject and interfere with the performance of cognitive tasks, possibly leading to results that are due to the side effects of stimulation and not caused by direct interference with brain function. However, this important methodological issue has not yet been assessed systematically.

In a pilot study, we monitored the influence of TMS

* Department of Psychiatry, University of Ulm, Germany.

+ Department of Radiology, University of Ulm, Germany.

^ Psychiatric University Hospital, Zürich, Switzerland.

Accepted for publication: March 15, 2005.

Correspondence and reprint requests should be addressed to $P D$ Dr. Uwe Herwig/Dr. Birgit Abler, Department of Psychiatry, University of Ulm, Leimgrubenweg 12, 89075 Ulm, Germany.

Fax: +49 73150026731

E-mails: uwe.herwig@puk.zh.ch

birgit.abler@medizin.uni-ulm.de

Copyright $\odot$ 2005, Springer Science + Business Media, Inc. side effects on task performance in the frame of a working memory study. In order to interfere with memory performance, TMS was applied over cortical areas that had been primarily identified by functional magnetic resonance imaging (fMRI). Subjective disturbance by TMS was recorded immediately after the stimulations. We analysed the correlation between task performance and the degree of disturbance.

\section{Methods}

Written informed consent was obtained from nine healthy right-handed subjects (age 23-33, four female) prior to their inclusion. The study was approved by the local ethics committee according to the Declaration of Helsinki. All participants took part in the fMRI trial, six of them underwent TMS.

The scope of this report is on the impact of stimulation side effects on task performance. Methods and results of the fMRI part as well as of a follow-up TMS trial are reported elsewhere (Herwig et al. 2003). We used a delayed match-to-sample-task with each trial consisting of set, delay and probe. In the task, six yellow letters ("set") were presented for $3500 \mathrm{~ms}$, followed by a delay of $6000 \mathrm{~ms}$, followed by a probe letter displayed for 1500 ms. Subjects determined whether the probe letter had been among the letters of the set. Reaction times and errors (incorrect answers given within the required time period of $1500 \mathrm{~ms}$ ) were registered. 


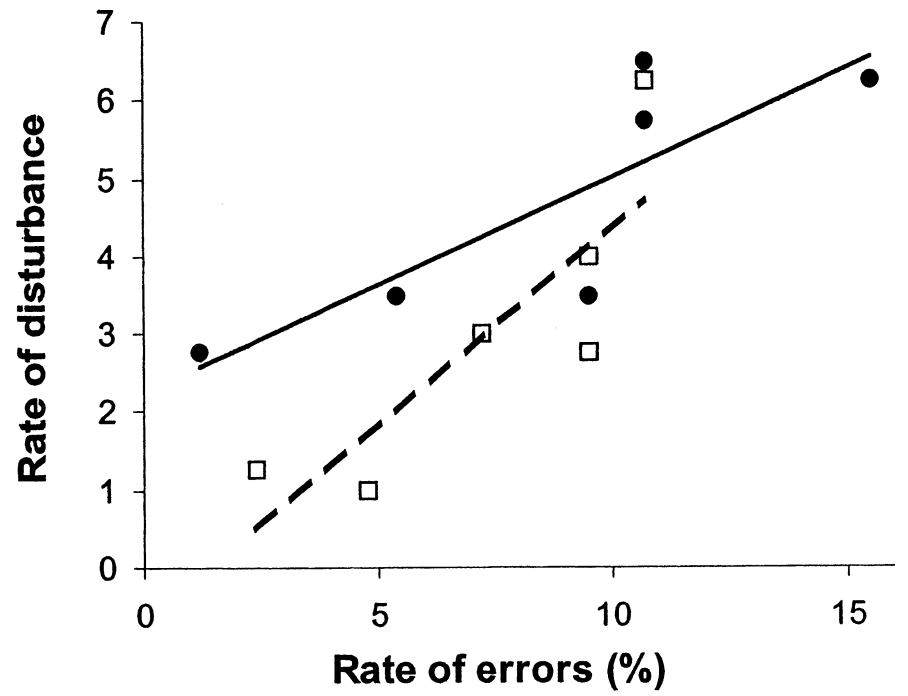

Figure 1. Correlation of mean rates of errors (x-axis) against mean rates of subjective disturbance by the uncomfortable sensation ( $y$-axis) from the TMS trials ( 4 frontal and 4 parietal stimulation blocks per subject) of the six subjects. Squares/dashed line ( $r=0.84)$ : parietal TMS; dots/straight line $(r=0.88)$ : frontal TMS.

TMS was applied using a MagPro Stimulator (Dantec/Medtronic, Germany) with a figure-of-eight coil (MC-B70) at a frequency of $15 \mathrm{~Hz}$ and with an intensity of $110 \%$ of the individual motor threshold (Rossini et al. 1994). The stimulation was applied during the second half of the $6000 \mathrm{~ms}$ delay (45 stimuli) to avoid exceeding safety criteria (Wassermann 1998).

A neuronavigational system $\left(\mathrm{STN}^{\mathrm{TM}}\right.$, Zeiss, Oberkochen) was used to guide the coil to the individually involved cortical areas (Herwig et al. 2002). These individual cortical sites were identified by $f M R I$ prior to the stimulations (Herwig et al. 2003). The subjects showed a left-lateralized network of activated areas in fMRI, including dorsolateral prefrontal, premotor, inferior frontal, parietal and temporo-parietal cortical regions. In each subject we selected for "Verum" stimulation those sites accessible to TMS in prefrontal and temporo-parietal cortex found in only one hemisphere (lateralized) with the most significant BOLD signal changes in fMRI. "Control" stimulation was applied above mirrored locations of the other hemisphere thus causing equal discomfort for active and control sites, as controlled by the questionnaires. For "sham" stimulation the coil was held in midline above the vertex without touching the skull at a distance that no longer caused any subjective tactile sensation. Prefrontal stimulation sites were selected individually within Brodmann area (BA) 9 in 5 subjects and its border to BA 6 in one subject. Parietal stimulation sites were in the area of the intra-parietal sulcus and the temporo-parietal junction (mainly BAs 40/7) in all six subjects that underwent TMS.

An experimental TMS-block comprised 21 trials. Each subject performed a total of 9 blocks of the task in a pseudo-randomized order: 2 blocks with stimulation of each of the 4 sites (left/right parietal, left/right prefrontal) and one block with sham stimulation. Wilcoxon's statistics were applied to compare behavioural data.

Immediately after each TMS block for each location, the subjects were asked to determine the degree of subjective disturbance caused by the stimulation with a questionnaire. On two scales from 1 (no disturbance) to 10 (strong disturbance) they indicated by integer i) how strongly they were disturbed by the noise, and ii) how strongly they were disturbed by the uncomfortable sensation on the skull.

Linear correlations (Spearman's rank correlation) between subjective rates of discomfort and wrong answers in the delayed match-to-sample task were assessed. Rates of discomfort were compared using as well the Wilcoxon signed rank test.

\section{Results}

Six of nine subjects participated in the TMS experiment. One subject refused TMS, one was excluded due to fMRI-artefacts, another because symmetrically bilateral activations made our control condition impossible.

In the subjects that underwent TMS we consistently found that the pooled (4 frontal and 4 parietal stimulation blocks per subject) mean rates of errors in the delayed match-to-sample-task and the pooled mean rates of disturbance by the sensation assessed by the questionnaire were linked: The more disturbed subjects felt by the painful sensation caused by stimulation, the more wrong answers they gave. There was a significant correlation between TMS side effects and error rates both in the parietal (Spearmans $\mathrm{r}=0.84 ; \mathrm{p}=0.04$ ) and in the frontal condition (Spearmans $\mathrm{r}=0.88 ; \mathrm{p}=0.02$ ) (figure 1).

Rates of errors in the delayed match-to-sample-task are shown in table I. More errors were made during stimulation of prefrontal "control" sites $(\mathrm{p}=0.003)$ than during sham. A similar trend was observed for stimulation of prefrontal "verum" sites $(\mathrm{p}=0.07)$ and for parietal "verum" stimulation ( $p=0.07)$, but not for parietal "control" $(\mathrm{p}=0.21)$, when compared with sham. No significant difference to sham was found when comparing reaction times. Further, the performance did not differ when comparing "verum" and "control" conditions in this six letter task.

The ratings of the side effects questionnaire (table II) for the disturbance caused by the sensation that repetitive TMS induces to the skull were significantly (Wilcoxon: $\mathrm{p}=0.03$ ) higher than the ratings for the noise 
Table I. Rates of errors, standard deviations (SD), and Wilcoxon statistics of verum and control stimulation (stim) at the four stimulation sites (mean of 2 blocks of TMS at each site) compared to sham stimulation (1 block). ": Significant difference to sham stimulation.

\begin{tabular}{lcc} 
Stimulation site & Rate of errors (SD) & $\begin{array}{c}\text { Stim vs sham } \\
\text { Wilcoxin p= }\end{array}$ \\
\hline Prefrontal "verum" & $8.3 \%(4.2)$ & 0.07 \\
Prefrontal "control" & $9.3 \%(7.1)^{*}$ & 0.003 \\
Parietal "verum" & $7.7 \%(4.9)$ & 0.07 \\
Parietal "control" & $6.8 \%(4.3)$ & 0.21 \\
Sham stimulation & $4.0 \%(5.6)$ & --
\end{tabular}

with a mean value of 3.9 of 10 points $(\mathrm{SD}=2.5)$. Particularly the frontal stimulation was associated with discomforting sensation and a high mean rate of disturbance of 4.7 points $(\mathrm{SD}=1.6)$. This was significantly (Wilcoxon: $\mathrm{p}=0.028)$ higher than the value for the parietal stimulation (3.0 points; $S D=1.9)$. There were no significant differences in the degree of subjective disturbance between verum and control sites, neither prefrontal nor parietal. Correlation analyses of reaction times and rates of disturbance showed no significant interaction. The results concerning the disturbance caused by the noise showed that subjects marked low values ( 2.5 of 10 points; $\mathrm{SD}=1.9)$ on the rating scale, with frontal $(2.8$ of $10 ; \mathrm{SD}=1.8)$ as well as with parietal TMS ( 2.3 of $10 ; \mathrm{SD}=1.1)$.

\section{Discussion}

The main finding of this study is a correlation between error rates in a working memory task and subjective discomfort caused by the sensational effects of magnetic stimulation. While there was no difference between stimulation of an active cortical area according to fMRI and its contralateral counterpart in this task, we detected increased error rates in the disturbing prefrontal stimulation condition compared to the non-painful sham stimulation. Disregarding the impact of side effects, our results would allow the assumption of a specific influence of TMS on cortical activity, and therefore could support the concept of an integration of the dorsolateral prefrontal cortex within the process of working memory during the delay of a working memory task. Several TMS studies (named on request) have reported similar results comparing a cortical stimulation with a non-painful sham stimulation and have interpreted these effects as the consequence of direct influence on cortical activation
Table II. Mean rates of disturbance concerning local sensation and noise at the four different stimulation sites. "Significant difference of sensation disturbance between pooled (control and verum) prefrontal and parietal stimulation (Wilcoxon $p=0.028$ ).

\begin{tabular}{lll} 
Stimulation site & \multicolumn{2}{c}{ Mean rates of disturbance (SD) } \\
& sensation & noise \\
\hline Prefrontal "verum" & $4.9(1.8)^{*}$ & $2.8(1.9)$ \\
Prefrontal "control" & $4.5(1.8)^{*}$ & $2.8(1.6)$ \\
Parietal "verum" & $3.1(1.9)$ & $2.3(1.1)$ \\
Parietal "control" & $3.0(2.0)$ & $2.4(1.1)$
\end{tabular}

by the administration of TMS.

However, the investigation of subjective sensational discomfort caused by the stimulation makes an alternative explanation possible, namely that the frontal stimulation effect is not due to specific interference with brain function but rather to the difference in discomfort between frontal and sham stimulation. Whereas participants hardly felt disturbed during the sham condition without local sensory perception, TMS was considered to cause a quite uncomfortable, bothersome local sensation. The sensations further were more disturbing when applied above prefrontal cortex than above parietal cortex, but comparable when stimulating right or left sided as verum and control conditions. This indicates that a mirrored control condition may be more appropriate to rule out a side effects bias on the results than a control condition above different cortex locations or even a sham condition. The local sensations were clearly more disturbing than the acoustic artefact of TMS. Side effects may be more pronounced when using TMS protocols like in this study with rTMS at a higher frequency and at an intensity above motor threshold. Nevertheless, any stimulation intended to cause neural depolarisation will cause side effects not present during a sham condition without touching the head or even a control task without stimulation.

In conclusion, the demonstrated correlation between the degree of discomfort due to TMS and the task performance indicates that subjective disturbance by the uncomfortable sensation may be an important confounding variable in TMS studies investigating functional brain mapping. It is therefore recommended to consider this fact in the design of future TMS studies, e.g. by choosing a similar discomforting control condition or by integrating subjective discomfort as a covariate of no interest in the analysis of TMS interference effects. 


\section{References}

Herwig, U., Koelbel, K., Wunderlich, A.P., Thielscher, A., von Tiesenhausen, C., Spitzer, M. and Schönfeldt-Lecuona, C. Spatial congruence of neuronavigated transcranial magnetic stimulation and functional neuroimaging. Clin. Neurophysiol., 2002, 113: 462-468.

Herwig, U., Abler, B., Schönfeldt-Lecuona, C., Wunderlich, A., Grothe, J., Spitzer, M. and Walter, H. Verbal storage in a premotor-parietal network: evidence from fMRI-guided magnetic stimulation. Neuroimage, 2003, 20: 1032-1041.

Rossini, P.M., Barker, A.T., Berardelli, A., Caramia, M.D., Caruso, G., Cracco, R.Q., Dimitrijevic, M.R., Hallett, M.,Katayama, Y., Lücking, C.H, Maertens de Noordhout, A.L, Marsden,
C.D., Murray, N.M.F., Rothwell, J.C., Swash, M. and Tomberg, C. Non-invasive electrical and magnetic stimulation of the brain, spinal cord and roots: basic principles and procedures for routine clinical application. Report of an IFCN committee. Electroencephalogr. Clin. Neurophysiol., 1994, 91: 79-92.

Walsh, V. and Cowey, A. Transcranial magnetic stimulation and cognitive neuroscience. Nat. Rev. Neurosci., 2000, 1: 73-79.

Wassermann, E.M. Risk and safety of repetitive transcranial magnetic stimulation: report and suggested guidelines from the International Workshop on the Safety of Repetitive Transcranial Magnetic Stimulation, June 5-7, 1996. Electroencephalogr. Clin. Neurophysiol., 1998: 1-16. 